\title{
Investigating strategies to improve attendance at screening visits in a randomized trial
}

\author{
Fang Chen, K Rahimi, R Haynes, K Naessens, M Taylor-Clarke, C Murray, Jane Armitage* \\ From Clinical Trials Methodology Conference 2011 \\ Bristol, UK. 4-5 October 2011
}

\begin{abstract}
Background
A common method of recruiting for randomized trials is to send letters to potentially eligible patients inviting them to a screening appointment. In 3 consecutive UK studies the proportion attending from those invited fell from $49 \%$ [1] in 1994-1997 and 42\%[2] in 1998-2001 to $13 \%$ at the beginning of an ongoing study in 2007. Procedures were similar in the 3 trials except that in 2007 the Patient Information Leaflet (PIL) was enclosed with the invitation letter. In order to understand whether the contents and/or style of invitation would explain the declining trend, 2 separate randomized comparisons were undertaken during the recruitment for the ongoing study.
\end{abstract}

\section{Methods}

Potentially eligible patients identified from hospital records were randomized to receive either an invitation letter enclosing the PIL (a 12-page A5 MREC approved booklet giving detailed information about the trial) or just a one page summary. A second comparison was made between a PIL modified after Focus Group discussions and the original PIL. Modifications included more colours, pictures and simplified language. The pre-specified endpoints for these assessments were the proportions of patients attending the screening visit, and entering the pre-randomization run-in period.

\section{Results}

Between July and October 2008, 20,759 personalized invitation letters were randomized to have the PIL or brief summary enclosed. There were no significant differences in either the proportions attending: PIL enclosed 1122/10,566 (10.6\%) versus not 1181/10,590

Clinical Trial Service Unit \& Epidemiological Studies Unit, University of Oxford, OX3 7LF, UK
(11.2\%) [OR 1.06; 95\%CI 0.97-1.16]; or in the proportion entering the pre-randomization run-in: $720 / 1181(6.8 \%$ of those invited) versus 690/1122 (6.5\%) [OR 1.05; (0.941.17)].

From November 2009 to January 2010, 12,164 patient invitations were randomized to enclose either the modified or the original PIL. A 17\% higher attendance was detected for the modified PIL: 580/6104 (9.5\%) versus $499 / 6060(8.2 \%)$ for the original PIL [OR=1.17; (1.031.33): $\mathrm{p}=0.01$ ). However there was no significant difference in the proportion entering the pre-randomization run-in: 373 (6.1\% of those invited) vs 339 (5.6\%) for modified versus original (OR 1.10; 0.94-1.28).

\section{Conclusion}

Whether the full PIL or brief summary was enclosed with the invitation did not affect the likelihood of attending or entering the run-in. Enclosing a more patient friendly PIL modestly improved the chance of attending, but not whether patients agreed to enter the study.

Published: 13 December 2011

\section{References}

1. MRC/BHF Heart Protection Study Collaborative Group: MRC/BHF Heart Protection Study of cholesterol-lowering therapy and of antioxidant vitamin supplementation in a wide range of patients at increased risk of coronary heart disease death: early safety and efficacy experience. Eur Heart J 1999, 20:725-741.

2. SEARCH Study Collaborative Group: Study of the effectiveness of additional reductions in cholesterol and homocysteine (SEARCH): characteristics of a randomized trial among 12064 myocardial infarction survivors. Am Heart J 2007, 154:815-823, e6.

doi:10.1186/1745-6215-12-S1-A111

Cite this article as: Chen et al:: Investigating strategies to improve attendance at screening visits in a randomized trial. Trials 2011 12(Suppl 1):A111. 